Latest Antipsychotic Drugs in Schizophrenia Study (CUtLASS 1). Arch Gen Psychiatry 2006; 63: 1079-87.

3 Jones PB, Barnes TRE, Elton P, Davies L, Dunn G, Lloyd H, et al. Firstvs second-generation antipsychotic drugs in schizophrenia - reply. Arch Gen Psychiatry 2007; 64: 979-80.

4 Soares BG, Fenton M, Chue P. Sulpiride for schizophrenia. Cochrane Database Syst Rev 2000; 2: CD001162.

5 Weizman A, Maoz B, Treves I, Asher I, Ben David M. Sulpiride-induced hyperprolactinemia and impotence in male psychiatric outpatients. Prog Neuropsychopharmacology Biol Psychiatry 1985; 9: 193-8.

6 Schoemaker H, Claustre Y, Fage D, Rouquier L, Chergui K, Curet O, et al. Neurochemical characteristics of amisulpride, an atypical dopamine D2/D3 receptor antagonist with both presynaptic and limbic selectivity. J Pharmaco Exp Ther 1997; 280: 183-97.

7 Glaser DN. The controversy of significance testing: misconceptions and alternatives. Am J Crit Care 1999; 8: 291-6.

Michael J. Peluso, MPhil, Yale School of Medicine, Harkness Hill, ESH 219, 367 Cedar Street, New Haven, CT 06510, USA. Email: michael.peluso@yale.edu; Shôn w. Lewis, MD, University of Manchester; Thomas R. E. Barnes, DSC, Centre for Mental Health, Imperial College London; Peter B. Jones, MD, PhD, Department of Psychiatry, University of Cambridge, and Early Intervention Services for Cambridgeshire \& Peterborough NHS Foundation Trust (CAMEO), UK

doi: 10.1192/bjp.201.3.247a

\section{Epidemiological challenges in systematic reviews}

We agree with Brugha and colleagues that the field of psychiatric epidemiology poses particular challenges to systematic reviewers. ${ }^{1}$ Heterogeneity between studies may arise from differences in outcomes and it is certainly true that psychiatry still lacks 'biologically based gold standards' regarding their definition. However, we disagree that these are necessarily linked. For the purpose of systematic reviews and meta-analyses, the issue is not to what extent an outcome is definable, with biological tests or otherwise; rather, how comparable individual studies are in their measurement of whatever outcome they use. For example, studies of schizophrenia defined by standard diagnostic tools such as the ICD-10, and applied using common operationalised criteria, should be looking at the same construct to a large extent. Definitions of physical health conditions also vary, even when specific tests are available for diagnosis. For example, definition of hypertension is not the same across national guidelines used in the USA and Europe. ${ }^{2,3}$ We acknowledge that differences exist in psychiatry between diagnostic tools which attempt to define the same or similar conditions, such as schizophrenia in ICD-10 $v$. DSM-IV. Often studies include outcomes such as psychotic, depressive or other symptoms instead of a diagnostic category, which can make comparison harder. Therefore, we recommend systematic reviews pay close attention to how outcome is defined in individual studies so that they are comparable. This should be considered as part of mandatory reporting of individual study quality in systematic reviews, as we have recently done, ${ }^{4}$ and as Brugha et al rightly encourage. Biologically based outcomes may help in due course but, currently, attention needs to be focused on the principle of comparability of outcomes we have now.

Another important contributor to heterogeneity is variation in exposure measurement which we think needs to be emphasised. In our systematic review and meta-analysis of premorbid IQ in schizophrenia, we found that the effect size varied as a result of differences in IQ testing methods and age at testing. ${ }^{5}$ Therefore, as well as ensuring that measurement of exposure is similar across included studies, differences should be explored further by subgroup and sensitivity analysis.

With regard to meta-analysis, combining methodologically incomparable studies will have serious implications for the validity and generalisability of findings. For example, a pooled odds ratio of 1.34 was reported for schizophrenia for exposure to herpes simplex virus type $2(\mathrm{HSV}-2)$ in a recent meta-analysis. ${ }^{6}$ Unfortunately, this tells us very little because the reviewers conflated studies which considered HSV-2 infection in early life and subsequent schizophrenia (i.e. prospective designs) with those which considered the prevalence of infection in people with established schizophrenia (i.e. a cross-sectional design). Such differences may not be picked up by tests for heterogeneity. The responsibility for establishing that individual studies are sufficiently comparable in design and other aspects in order to justify combining their results in a meta-analysis lies with researchers conducting systematic reviews, as well as with the reader.

It was not clear from the meta-review how many original reviews followed some kind of guidelines. Guidelines for reporting of systematic reviews, including those of observational studies, already exist, such as Preferred reporting Items for Systematic reviews and Meta-Analyses (PRISMA) and Meta-analyses Of Observational Studies in Epidemiology (MOOSE). They include comprehensive checklists for the assessment, for example, of outcome, exposure, effects of bias and confounding in individual studies. We believe more widespread use of these guidelines, something that can be mandated by journal editors and peer reviewers, should greatly increase comparability of individual studies, and overall, lead to an improvement in the quality of systematic reviews and meta-analyses.

1 Brugha TS, Matthews R, Morgan Z, Hill T, Alonso J, Jones DR. Methodology and reporting of systematic reviews and meta-analyses of observational studies in psychiatric epidemiology: systematic review. Br J Psychiatry 2012; 200: 446-53.

2 Chobanian AV, Bakris GL, Black HR, Cushman WC, Green LA, Izzo Jr JL, et al. The Seventh Report of the Joint National Committee on Prevention, Detection, Evaluation, and Treatment of High Blood Pressure: the JNC 7 report. JAMA 2003; 289: 2560-72.

3 Mancia G, De Backer G, Dominiczak A, Cifkova R, Fagard R, Germano G, et al. 2007 Guidelines for the management of arterial hypertension: The Task Force for the Management of Arterial Hypertension of the European Society of Hypertension (ESH) and of the European Society of Cardiology (ESC). Eur Heart J 2007; 28: 1462-536.

4 Kirkbride JB, Errazuriz A, Croudace TJ, Morgan C, Jackson D, Boydell J, et al. Incidence of schizophrenia and other psychoses in England, 1950-2009: a systematic review and meta-analyses. PLOS One 2012; 7: e31660.

5 Khandaker GM, Barnett JH, White IR, Jones PB. A quantitative meta-analysis of population-based studies of premorbid intelligence and schizophrenia. Schizophr Res 2011; 132: 220-7.

6 Arias I, Sorlozano A, Villegas E, de Dios Luna J, McKenney K, Cervilla J, et al. Infectious agents associated with schizophrenia: a meta-analysis. Schizophr Res 2012; 136: 128-36.

Golam M. Khandaker, Wellcome Trust Clinical Research Fellow, EpiCentre Group, Department of Psychiatry, University of Cambridge, UK. Email: gmk24@medschl.cam.ac.uk; James B. Kirkbride, Peter B. Jones, Department of Psychiatry, University of Cambridge, UK

doi: 10.1192/bjp.201.3.248

\title{
Correction
}

Impact of ethnic density on adult mental disorders: narrative review. BJP, 201, 11-19. Reference 48 should read: Schofield P,
Ashworth M, Jones R. Ethnic isolation and psychosis: re-examining the ethnic density effect. Psychol Med 2011; 41: 1263-9. 